\title{
Review of: "Health Risk Evaluation for Fluoride and Nitrate and A Baseline Study of Uranium in Hard Rock Aquifers of Northern Karnataka, India"
}

Anil Kumar Haritash

Potential competing interests: The author(s) declared that no potential competing interests exist.

Not in the shape of a scientific manuscript.

The authors are advised to study good research papers related to the subject.

It is not fit for publication, in my opinion. 